\title{
Targeted inhibition of STAT3 in neural stem cells promotes neuronal differentiation and functional recovery in rats with spinal cord injury
}

\author{
TINGTING LI ${ }^{1 *}$, XIAOYANG ZHAO ${ }^{2 *}$, JING DUAN $^{3}$, SHANGBIN CUI ${ }^{2}$, \\ KAI ZHU ${ }^{2}$, YONG WAN ${ }^{2}$, SHAOYU LIU ${ }^{2}$, ZHIMING PENG ${ }^{4}$ and $\mathrm{LE} \mathrm{WANG}^{2}$ \\ ${ }^{1}$ Department of Rehabilitation Medicine, The First Affiliated Hospital of Sun Yat-sen University, Guangzhou, Guangdong \\ 510700; Departments of ${ }^{2}$ Spine Surgery and ${ }^{3}$ Pathology, The First Affiliated Hospital of Sun Yat-sen University, \\ Guangzhou, Guangdong 510080; ${ }^{4}$ Department of Orthopaedics, Air Force General Hospital, Beijing 100142, P.R. China
}

Received September 19, 2020; Accepted February 2, 2021

DOI: $10.3892 /$ etm.2021.10143

\begin{abstract}
STAT3 is expressed in neural stem cells (NSCs), where a number of studies have previously shown that STAT3 is involved in regulating NSC differentiation. However, the possible molecular mechanism and role of STAT3 in spinal cord injury (SCI) remain unclear. In the present study, the potential effect of STAT3 in NSCs was first investigated by using short hairpin RNA (shRNA)-mediated STAT3 knockdown in rat NSCs in vitro. Immunofluorescence of $\beta 3$-tubulin and glial fibrillary acidic protein staining and western blotting showed that knocking down STAT3 expression promoted NSC neuronal differentiation, where the activity of mTOR was upregulated. Subsequently, rats underwent laminectomy and complete spinal cord transection followed by transplantation of NSCs transfected with control-shRNA or STAT3-shRNA at the injured site in vivo. Spinal cord-evoked potentials and the Basso-Beattie-Bresnahan scores were used to examine functional recovery. In addition, axonal regeneration and tissue repair were assessed using retrograde tracing with FluoroGold, hematoxylin and eosin, Nissl and immunofluorescence staining of $\beta 3$-tubulin, glial fibrillary acidic protein and microtubule-associated protein 2 following SCI. The results showed that transplantation with NSCs transfected with STAT3-RNA enhanced functional recovery following SCI
\end{abstract}

Correspondence to: Dr Le Wang, Department of Spine Surgery, The First Affiliated Hospital of Sun Yat-sen University, 58 Zhongshan 2nd Road, Guangzhou, Guangdong 510080, P.R. China

E-mail:wangle3@mail.sysu.edu.cn

Dr Zhiming Peng, Department of Orthopaedics, Air Force General Hospital, 30 Fucheng Road, Beijing 100142, P.R. China

E-mail: zhiming1150101@hotmail.com

*Contributed equally

Key words: STAT3, spinal cord injury, neural stem cells, neuronal differentiation and promoted tissue repair in rats, in addition to improving neuronal differentiation of the transplanted NSCs in the injury site. Taken together, in vitro and in vivo evidence that inhibiting STAT3 could promote NSC neuronal differentiation was demonstrated in the present study. Therefore, transplantation with NSCs with STAT3 expression knocked down appears to hold promising potential for enhancing the benefit of NSC-mediated regenerative cell therapy for SCI.

\section{Introduction}

Spinal cord injury (SCI) frequently causes persistent functional deficits due to the absence of spontaneous axon regeneration, formation of large cavities and glial scars that interrupt the ascending and descending neural pathways $(1,2)$. In rodent models of SCI, levels of pro-inflammatory interleukins (ILs), such as IL-6, peak acutely in the injured areas and lead to the activation of the Janus kinase 1-signal transducer and STAT3 signaling pathway (3). STAT3 signaling is also upregulated in a number of neurodegenerative diseases. For instance, in patients with amyotrophic lateral sclerosis, the spinal cord microglia, reactive astrocytes and motor neuron nuclei were all shown to exhibit increased levels of phosphorylated (p-) STAT3 (p-STAT3) (4). Another study previously reported that STAT3 is an important signaling component during reactive astrogliosis through the Notch 1-STAT3-endothelin receptor type B signaling axis (5). A growing body of evidence suggests that STAT3 is an injury-induced signaling mechanism that is critical for various aspects of nerve regeneration (6-8). In addition, in vitro suppression of STAT3 (9) or its conditional deletion in vivo (10) have been demonstrated to induce neurogenesis and inhibit astrogliosis. Therefore, STAT3 appears to be the key to enhancing neurogenesis following SCI.

Neural stem cell (NSC) transplantation has been demonstrated to be a promising treatment method for improving tissue repair and functional recovery following SCI (11). Mechanistically, NSCs may differentiate into neurons and glial cells to bridge the damaged area, re-establish conduction pathway, form functional synapses by expressing genes associated with axonal regeneration and by secreting neurotrophic 
growth factors in the injured spinal cord (12). However, accumulating evidence has suggested that NSC-based therapy cannot achieve optimal results, mainly due to the limited differentiation potential of neurons and excessive differentiation of astrocytes, which contributes to glial scarring (13). Natarajan et al (14) demonstrated that treatment with an inhibitor of STAT3 promoted NSC differentiation into neurons and suppressed glial differentiation in vitro, a finding that was also subsequently confirmed by White et al (15). However, few studies have assessed the effects of STAT3 silencing on NSCs transplantation and its functional outcomes downstream in vivo.

In the present study, the effects of STAT3 RNA interference (RNAi) on NSC survival and differentiation in vitro and the potential mechanisms underlying these effects were first explored. NSCs with STAT3 expression were the knocked down before being injected into the injury site of a rat SCI model to investigate its effects on neurodegeneration in vivo. The focus was placed on exploring the effect of transplanting STAT3-silenced NSCs on spinal cord injury to provide novel targets for the treatment and rehabilitation of SCI in the future.

\section{Materials and methods}

Lentiviral vector construction. Green fluorescent protein (GFP) was also encoded by the lentiviral vector with a sequence that specifically silenced the STAT3 gene [STAT3-short hairpin RNA (shRNA); Shanghai GeneChem Co., Ltd.]. The oligonucleotides were synthesized and ligated into the GV248 plasmid-Hu6-MCS-Ubiquitin-IRES-puromycin plasmid (cat. no. PC0NGC248028254; Shanghai GeneChem Co., Ltd.) and had the following STAT3-shRNA sequence: 5'-CAGCAG ATGCTGGAACAGCAT-3'. A nontargeting sequence (5'-TTC TCCGAACGTGTCACGT-3') was also carried by a control lentivirus (LV) vector. The LV particles were generated as previously described (16) to a final titer of $1 \times 10^{8} \mathrm{TU} / \mathrm{ml}$.

NSC isolation and culture. 3-month old pregnant Sprague-Dawley rats (Laboratory Animal Center of Sun Yat-sen University; $n=3$ ) were sacrificed and NSCs were extracted from the brain tissues of the fetuses $(n=14)$ on embryonic day 14 as previously described (17). The animals were housed three to a cage with free access to food/water and kept under standardized atmosphere (temperature, $22^{\circ} \mathrm{C}$; humidity, 55\%; 12/12 h light/dark cycle). Rats were anesthetized with $1 \%$ pentobarbital sodium $(40-45 \mathrm{mg} / \mathrm{kg}$ ) to minimize pain and sacrificed by $\mathrm{CO}_{2}$ with the displacement rate at $20 \% / \mathrm{min}$ ). Fetal brain tissue was mechanically cut and removed in Hanks' balanced salt solution (Beijing Solarbio Science \& Technology Co., Ltd.), following which the cell suspension was centrifuged at $300 \mathrm{x} \mathrm{g}$ and $4^{\circ} \mathrm{C}$ for $5 \mathrm{~min}$. The supernatant was discarded and the cell pellet was diluted into DMEM/F-12 (Thermo Fisher Scientific, Inc.) as a single-cell suspension. NSCs were then plated in a T25 culture flask (Corning, Inc.) containing DMEM/F-12 (Thermo Fisher Scientific, Inc.) nutrient mixture, $1 \%$ L-glutamine (Thermo Fisher Scientific, Inc.), $2 \% \mathrm{~B} 27,1 \%$ penicillin/streptomycin, $20 \mathrm{ng} / \mathrm{ml}$ fibroblast growth factor-2 (FGF2; PeproTech, Inc.) and $20 \mathrm{ng} / \mathrm{ml}$ epidermal growth factor (EGF; PeproTech, Inc.). NSCs were cultured in $5 \% \mathrm{CO}_{2}$ at $37^{\circ} \mathrm{C}$ and passaged weekly by digesting with Accutase (EMD Millipore) in the aforementioned medium. NSCs at passages 2-4 were used for subsequent experiments $(15,16)$.

NSC transfection and differentiation. In the DMEM/F12 nutrient mixture, NSCs form neurospheres spontaneously. For cell transfection, 2nd passage neurospheres were dissociated into a single-cell suspension at a density of $1 \times 10^{5}$ cells $/ \mathrm{ml}$ and plated onto coverslips coated with $0.01 \%$ poly-L-lysine (Merck KGaA) in 12-well plates (Corning, Inc.). The aforementioned culture medium was then added to the 12-well plate to rejuvenate the cells. Cells were subsequently divided into the following three groups: No transfected NSCs group (CL, n=5), Lv-control shRNA transfected NSCs group (LV-CL, n=5), Lv-STST3 shRNA transfected NSCs group (LV-STAT3, $\mathrm{n}=5$ ). The medium was then changed to fresh medium after $24 \mathrm{~h}$. After $72 \mathrm{~h}$ transfection, GFP expression was visualized by fluorescence microscopy (x40 magnification; AG Axio Observer Z1; Zeiss AG). To quantify the suppressive effects of RNAi on STAT3 expression, STAT3 expression in each group was examined by reverse transcription-quantitative PCR (RT-qPCR) and western blotting. To assess the differentiation capacity of NSCs, the culture medium was changed to the differentiation medium (culture medium without growth factors EGF and FGF2) for 1 week after transfection.

Western blotting. Protein lysates (RIPA Lysis and Extraction Buffer; cat. no. 89901; Thermo Fisher Scientific, Inc.) were extracted from the cells used in the in vitro study ( $\mathrm{n}=5$ per group, harvested 3 days after transfection). Bicinchoninic acid assay (Beyotime Institute of Biotechnology) was used to measure protein concentration, which was equilibrated prior to loading. Protein samples in each group were separated by SDS-PAGE (10\% Bis-Tris gel), transferred onto PVDF membranes (EMD Millipore) and blocked with 5\% BSA (Merck $\mathrm{KGaA}$ ) for $1 \mathrm{~h}$ at $24^{\circ} \mathrm{C}$, followed by incubation with primary antibodies at $4^{\circ} \mathrm{C}$ overnight. Primary antibodies targeting STAT3 (cat. no. ab68153; Abcam), p-STAT3 (cat. no. 9145; Cell Signaling Technology, Inc.), mTOR (cat. no. 2983; Cell Signaling Technology, Inc.), p-mTOR (cat. no. 2971; Cell Signaling Technology, Inc.) and GAPDH (cat. no. 5174; Cell Signaling Technology, Inc.) were used at a 1:1,000 dilution. After washing the membranes in Tris- $\mathrm{HCl}$ buffer containing $0.2 \%$ Tween-20 (TBST; pH 7.5) twice, the membranes were incubated with a filtered and horseradish peroxidase-conjugated goat anti-rabbit IgG antibody (1:5,000; cat. no. ab6721; Abcam) for $1 \mathrm{~h}$ at room temperature and then washed three times in TBST. Finally, the bands were visualized using enhanced chemiluminescence development system (EMD Millipore). For analysis of the Western blot images Image 1.50 (National Institutes of Health) was used.

$R N A$ extraction and $R T-q P C R$. Total cell RNA ( $\mathrm{n}=5$ per group, harvested 3 days after transfection) was extracted using TRIzol $^{\circledR}$ reagent (Invitrogen; Thermo Fisher Scientific, Inc.). RNA was reverse transcribed into cDNA using a reverse transcription system (GoScript ${ }^{\mathrm{TM}}$ Reverse Transcription System; cat. no. A5000; Promega Corporation). The process used was as follows: Combine RNA and cDNA primers at $70^{\circ} \mathrm{C}$ for $5 \mathrm{~min}$, 
followed by adding reverse transcription mix into primer/RNA mix at $25^{\circ} \mathrm{C}$ for $5 \mathrm{~min}, 42^{\circ} \mathrm{C}$ for $60 \mathrm{~min}$ and $70^{\circ} \mathrm{C}$ for $15 \mathrm{~min}$. qPCR was performed on an ABI 7900 PCR detection system (Thermo Fisher Scientific, Inc.) using SYBR ${ }^{\mathrm{TM}}$ Green PCR Master Mix (cat. no. 4309155, Thermo Fisher Scientific, Inc.). The process used was as follows: $95^{\circ} \mathrm{C}$ for $5 \mathrm{~min}$, followed by 40 cycles $\left(95^{\circ} \mathrm{C}\right.$ for $10 \mathrm{sec}$ and $60^{\circ} \mathrm{C}$ for $\left.30 \mathrm{sec}\right)$. Parallel amplification of the GAPDH housekeeping gene was used to normalize gene expression. PCR primer sequences are listed below: STAT3 forward, 5'-AATATAGCCGATTCCTGCAAG AG-3' and reverse, 5'-TGGCTTCTCAAGATACCTGCTC-3' and GAPDH forward, 5'-TGACGCTGGGGCTGGCATTG-3' and reverse, 5'-GGCTGGTGGTCCAGGGGTCT-3'. The relative expression level of target mRNA was calculated using the $2^{-\Delta \Delta C q}$ method (18).

Surgical procedures and cell transplantation. All experimental animal procedures were approved by the Care and Use Committee of Sun Yat-Sen University (approval no. SYXX2012-0081; November 9, 2016) and performed following the Guide for the Care and Use of Experimental Animals provided by the National Research Council (1996, USA) (19). All the animals were housed three to a cage with free access to food and water, where they were kept under a standardized atmosphere (temperature, $22^{\circ} \mathrm{C}$; humidity, 55\%; 12-h light/dark cycle).

Spinal cord surgery was performed on 60 adult female SD rats (weight range 200-250 g laboratory Animal Center of Sun Yat-sen University). All rats were healthy and did not undergo any previous procedures. The rats were randomly divided into the following four groups: i) Sham (spinal cord exposure only, $\mathrm{n}=10)$; ii) SCI $(\mathrm{n}=15)$; iii) STAT3-shRNA-transfected NSC (LS group, $n=20$ ); and iv) Control-shRNA-transfected NSC (LC group, $n=15$ ). In the Sham group, the rats underwent laminectomy without transection of the spinal cord. In the SCI group, rats underwent surgery with complete transection of the spinal cord. In the LC group, NSCs transfected with control LV were injected immediately into the rats following complete transection of the spinal cord. In the LS group, NSCs transfected with STAT3 LV were injected immediately into the rat's complete transection of the spinal cord. Briefly, animals were intraperitoneally injected with $1 \%$ sodium pentobarbital $(40-45 \mathrm{mg} / \mathrm{kg}$ ) for anesthesia. Laminectomy was then performed at the level of the 10th thoracic vertebra (T10). Next, the spinal cord was cut twice using scissors (once caudal to T10 and once rostral to T10) to achieve complete transection, following which a 2-mm block of the spinal cord was removed. Following hemostasis, the rats were injected with $5 \mu \mathrm{l}$ control-shRNA-transfected NSCs or STAT3-shRNA-transfected NSCs at a density of $1 \times 10^{5}$ cells $/ \mu 1$. Two injections were performed at the depth of $1 \mathrm{~mm}$ rostral, as well as $1 \mathrm{~mm}$ caudal to the injured site using a microsyringe at a rate of $0.5 \mu \mathrm{l} / \mathrm{min}$. Finally, following hemostasis, 5-0 sutures were used to suture the muscle and skin. To prevent infection and dehydration, $1 \mathrm{ml}$ saline containing $1 \times 10^{5}$ units of penicillin was intraperitoneally injected daily for 1 week (20). The postsurgical care of SCI rats included massaging the urinary bladder twice a day for urination until bladder function was restored. Surgery was performed in a blinded manner. At the 7th week after surgery,
5 rats were randomly selected from each group for retrograde tracking experiments [injection of Fluorogold (FG)]. After the experiment was completed, the rats were not sacrificed and continued to be raised for 1 week. On the 8th week, 5 rats were randomly selected from each group for the SCEP recording experiment. After the SCEP recording experiment, rats were anesthetized with $1 \%$ pentobarbital sodium (40-45 mg/kg) and then sacrificed by $\mathrm{CO}_{2}$ inhalation, using a displacement rate of $20 \% / \mathrm{min}$, for $H \& E$ staining $(n=5$ per group), fluorescent immunohistochemistry ( $\mathrm{n}=5$ per group) and neuron retrograde tracing staining ( $n=5$ per group).

Basso-Beattie-Bresnahan (BBB) test. The BBB locomotor rating scale is considered to be a reliable tool for evaluating impairment and recovery of motor abilities in hindlimbs following spinal cord injury (21) A total score of 21 points indicate that the locomotor ability had not been affected, while 0 points represents a severe deficit. The rats were placed in an open experimental field and allowed to move freely for $5 \mathrm{~min}$. During this period, the lower limb movement ability of the rats was observed and the movement of each rat was scored based on the BBB score scale. Hindlimb motor behavior was evaluated weekly for 8 weeks, with tests performed at the same time each day and grading performed by two investigators (XZ and JD) blinded to grouping.

Spinal cord-evoked potential (SCEP) recording. At 8 weeks post-injury, 20 rats ( $n=5$ in each group) were anesthetized with $1 \%$ pentobarbital sodium $(40-45 \mathrm{mg} / \mathrm{kg})$ and stereotaxically fixed. The T5-L1 vertebrae were then completely exposed. Finally, a stimulation electrode was inserted into the T5-T6 interspinous ligaments, following which a pair of needle electrodes were inserted into the interspinous ligaments of T12-L1 for SCEP recording. The electrodes were connected to a BL-420N series biological signal acquisition and analysis system (Chengdu Thai UNITA Co., Ltd.). The parameters of the SCEP signals were set as follows: i) Gain, 2,000; ii) Time constant, $0.01 \mathrm{sec}$; and iii) Filtering, $300 \mathrm{~Hz}$. To elicit a SCEP, a single-pulse stimulation $(50 \mathrm{msec}$ in duration at a frequency of $5.1 \mathrm{~Hz}$ and a voltage increase of $1 \mathrm{mV}$ ) was transmitted through the electrodes until a mild twitch of the vertebral body of the animal was observed. To obtain high-quality waveforms for the SCEP signals, 100 SCEP responses per rat were averaged.

Retrograde axonal tract tracing. Animals ( $\mathrm{n}=5$ per group) were submitted for neuron retrograde tracing using 4\% FG (cat. no. sc-358883; dilution, 1:25; Santa Cruz Biotechnology, Inc.) 7 weeks after SCI. Briefly, a dorsal laminectomy was performed under anesthesia $(40-45 \mathrm{mg} / \mathrm{kg}$ pentobarbital) at T12 before $0.5 \mu \mathrm{l} \mathrm{FG}$ was injected into the spinal cord using a microsyringe. A week after injection, the animals were perfused after $\mathrm{CO}_{2}$ euthanasia and the $\mathrm{T} 7$ segment of the spinal cord was collected to detect FG-labelled neurons (22).

Perfusion and cryosection. Rats were subjected to anesthesia with $1 \%$ pentobarbital sodium $(40-45 \mathrm{mg} / \mathrm{kg}) 8$ weeks after spinal cord surgery and sacrificed by $\mathrm{CO}_{2}$ using a displacement rate of $20 \% / \mathrm{min}$. Rats were then perfused transcardially with $0.9 \%$ normal saline and $400 \mathrm{ml} 4 \%$ paraformaldehyde 
(PFA). T8-L1 segments of the spinal cord were removed from rats without Fluorogold (FG) labelling and T7-L1 segments were removed from Fluorogold labelled rats. These segments were then fixed with $4 \%$ PFA at room temperature overnight and transferred to $30 \%$ sucrose for cryoprotection for 2-3 days at $4^{\circ} \mathrm{C}$ following collection. Tissue were embedded into OCT Tissue-Tek (cat. no. 4583; Sakura Finetek USA, Inc.). Embedded tissues were then sliced transversely or longitudinally at a thickness of $10 \mu \mathrm{m}$, mounted onto polylysine-coated glass slides and stored at $-20^{\circ} \mathrm{C}$ to be used in subsequent experiments.

Histopathological analysis. To assess the cavity area in the spinal cord, rats were sacrificed for H\&E staining 8 weeks after SCI. OCT Component (Tissue-Tek Frozen Embedding Agent; cat. no. 4583; Sakura)-embedded T8-T11 longitudinal spinal cord sections from each group were fixed in $4 \%$ paraformaldehyde for $30 \mathrm{~min}$ at room temperature, rehydrated in a graded series of ethanol (100\% twice, $95 \%$ twice, $90 \%$ and $85 \%$ for $5 \mathrm{~min}$ at room temperature), then the tissue was treated with hematoxylin solution for $5 \mathrm{~min}$ and eosin solution (cat. no. C0105S; Beyotime Institute of Biotechnology) for $1 \mathrm{~min}$ for $\mathrm{H} \& \mathrm{E}$ staining at room temperature or treated with Nissl solution (cat. no. C0117; Beyotime Institute of Biotechnology) for $5 \mathrm{~min}$ for Nissl staining at room temperature, rehydrated in the graded series of ethanol and xylene $(50 \%, 100 \%$ twice for $1 \mathrm{~min})$ at room temperature and observed using a light microscope at x20 magnification. The cavity area of these images was evaluated using NIH ImageJ software (1.50; National Institutes of Health).

Fluorescence immunohistochemistry. Frozen sections of spinal cord tissue sections or cells were fixed in 4\% PFA for $30 \mathrm{~min}$ and permeabilised with $0.3 \%$ Triton $\mathrm{X}-100$ for $30 \mathrm{~min}$, both at $4^{\circ} \mathrm{C}$. Blocking was performed with $5 \%$ normal goat serum (cat. no. C0265; Beyotime Institute of Biotechnology) for $1 \mathrm{~h}$ at $4^{\circ} \mathrm{C}$ and tissue sections were incubated with primary antibodies targeting the following proteins at $4^{\circ} \mathrm{C}$ overnight: Nestin (dilution, 1:200; cat. no. 33475; Cell Signaling Technology, Inc.), $\beta 3$-tubulin (dilution, 1:200; cat. no. 5568; Cell Signaling Technology, Inc.), GFAP (dilution, 1:300; cat. no. 80788; Cell Signaling Technology, Inc.) and microtubule-associated protein 2 (MAP2; dilution, 1:200; cat. no. 8707; Cell Signaling Technology, Inc.). Goat anti-rabbit IgG $(\mathrm{H}+\mathrm{L})$ Cross-Adsorbed Secondary Antibody, Alexa Fluor 555 (cat. no. A-21428; Thermo Fisher Scientific, Inc.), was used at a concentration of $2 \mu \mathrm{g} / \mathrm{ml}$ in PBS containing $0.2 \%$ BSA for $45 \mathrm{~min}$ at room temperature. ProLong Gold antifade reagent containing DAPI (dilution: 1:5000; cat. no. D1306; Thermo Fisher Scientific, Inc.) was used for nuclear staining at room temperature in spinal cord tissue sections. The total area of target marker-positive cells was then evaluated in each visual field under fluorescence (x40 magnification; Carl Zeiss Axio Observer Z1; Carl Zeiss AG) using ImageJ software (1.50, National Institutes of Health). A total of five random fields per section and five sections per group were examined independently by two observers (XZ and JD) blinded to grouping. The percent of $\beta 3$-tubulin or GFAP-positive cells was calculated by dividing the number of $\beta 3$-tubulin or GFAP-positive cells by the total number of cells.
Statistical analysis. All statistical analyses were performed using GraphPad Prism 6 software (GraphPad Software, Inc.). Shapiro-Wilk test was first used to evaluate the normality of all data in the present study. Experiments were repeated 5 times in vitro study, All data are expressed as the mean \pm SD and analyzed using one-way analysis of variance followed by Bonferroni post hoc tests for multiple comparisons or Student t-test for pairwise comparisons. $\mathrm{P}<0.05$ was considered to indicate a statistically significant difference.

\section{Results}

Knockdown of STAT3 promotes neuronal differentiation in NSCs. NSCs were passaged and allowed to grow for an additional 3-5 days to form a 100- $\mu$ m-diameter neurosphere from single cells. Immunostaining of the neurospheres showed that nestin, a surface marker of neural stem and precursor cells (23), was strongly expressed in the neurosphere (Fig. 1A). To achieve a specific knockdown of the STAT3 gene in NSCs, NSCs were transfected with STAT3 shRNA-expressing LV. GFP expression was observed by fluorescence microscopy $72 \mathrm{~h}$ after LV infection. Western blotting showed that STAT3 expression was significantly lower in the LV-STAT3 group compared with that in the LV-CL group (Fig. 1B and C). This observation was also confirmed by RT-qPCR analysis (Fig. 1D). Immunofluorescence staining analysis showed that the targeted inhibition of STAT3 induced the differentiation of NSCs into neurons, as the neuronal marker $\beta 3$-tubulin (24) was found to be expressed more frequently in the LV-STAT3 group compared with that in the CL and LV-CL groups. However, GFAP, a specific marker of astrocytes (23), was expressed at significantly lower levels in the LV-STAT3 group compared with those in the other two groups (Fig. 1E-G). These results suggest that a specific knockdown of STAT3 gene expression was achieved in NSCs, which supports the hypothesis that targeted inhibition of STAT3 promotes neuronal differentiation in NSCs, consistent with data from a previous study.

Targetedinhibition of STAT3 promotes mTOR activation.mTOR signaling is important for the maintenance and differentiation of NSC development, where the activation of mTOR promotes the involvement of NSCs in neurogenesis (25). To explore the relationship between the mTOR and STAT3 signaling pathways in NSC differentiation, the STAT3 signaling pathway was inhibited by treating NSCs that were transfected with shRNA-expressing LV. NSCs were cultured in a differentiation medium following transfection. On day 7 , western blotting was performed to examine the expression of STAT3 and mTOR. The expression of STAT3 itself and STAT3 phosphorylation was significantly lower in the LV-STAT3 group compared with that in the LV-CL group. Of note, although no difference in total mTOR expression was observed among the three groups, the levels of the activated form of mTOR (p-mTOR) was significantly higher in the LV-STAT3 group, compared with that in the LV-CL groups (Fig. 2A and B). These results suggest that STAT3 inhibition activated the mTOR signaling pathway, which may promote neuronal differentiation of NSCs.

Transplantation of STAT3-RNAi-transfected NSCs enhances functional recovery following SCI. To investigate if 

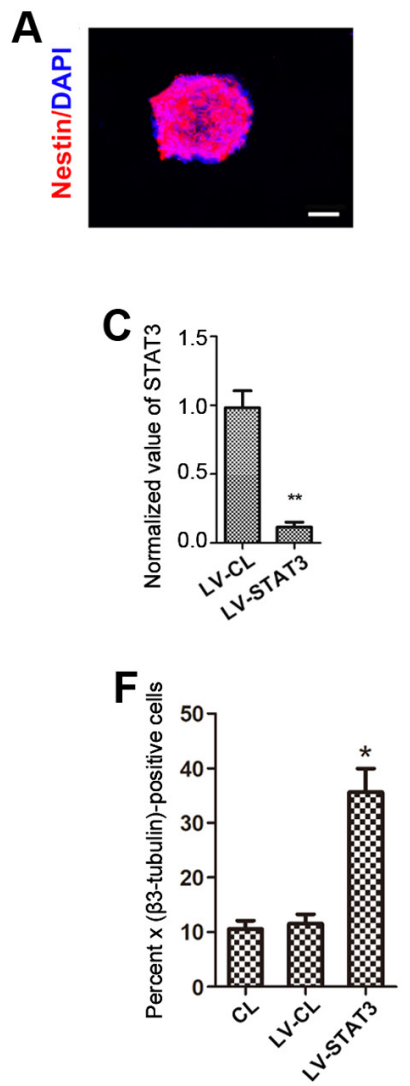

B

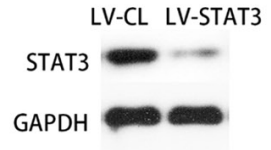

D

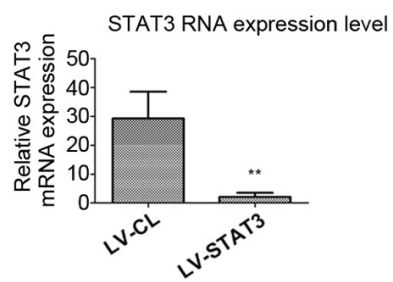

$\mathbf{G}$

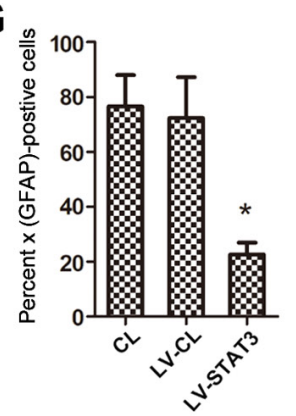

E
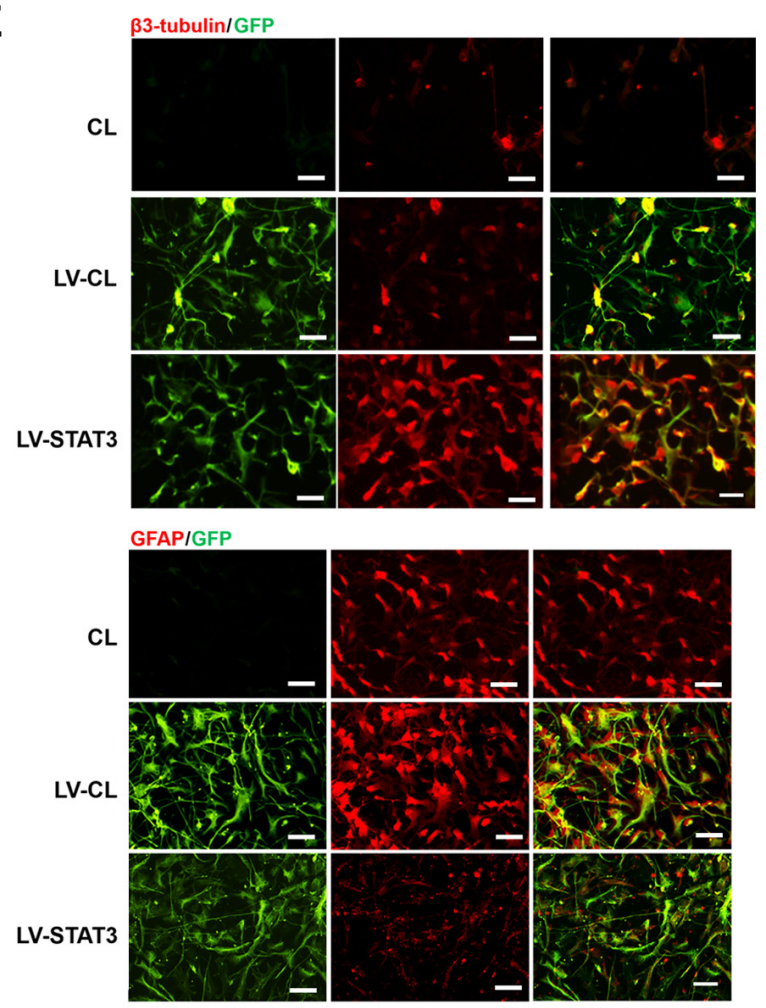

Figure 1. STAT3 knockdown promotes neuronal differentiation in NSCs. (A) Immunofluorescence indicated that the NSC-specific marker nestin was highly expressed in cells. (B and C) Western blotting was performed to measure STAT3 protein levels in each group. (D) Effect of STAT3 gene silencing on STAT3 mRNA expression measured using reverse transcription-quantitative PCR. (E) Immunostaining for $\beta 3$-tubulin and GFAP (red), GFP (green) in each group. Quantification of the percentage of (F) $\beta 3$-tubulin- and (G) GFAP-positive cells in each group. Scale bar, $50 \mu \mathrm{m}$. ${ }^{*} \mathrm{P}<0.05$ and ${ }^{* *} \mathrm{P}<0.01 \mathrm{vs}$. LV-CL. NSCs, neural stem cells; GFP, Green fluorescent protein; GFAP, fibrillary acidic protein; LV, lentivirus; CL, control.
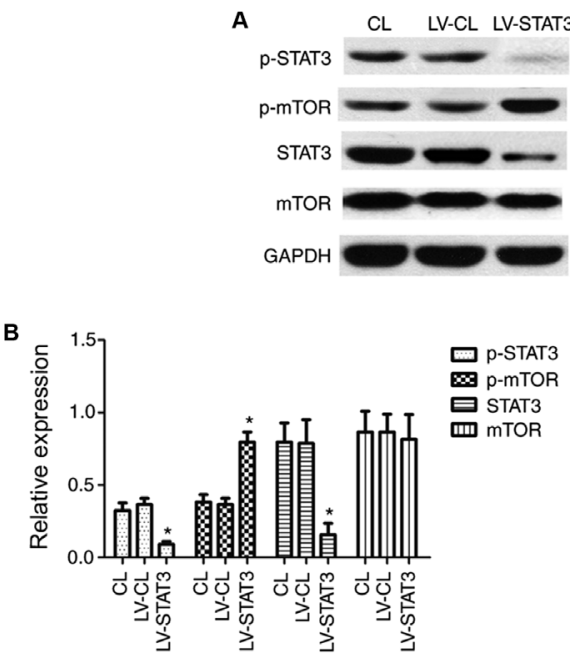

C

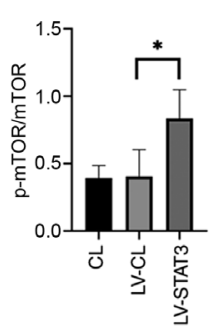

Figure 2. Targeted inhibition of STAT3 promotes mTOR activation. (A) Western blotting of STAT3, mTOR, p-STAT3 and p-mTOR in each group. (B) Quantification of western blotting for STAT3 and mTOR expression, in addition to STAT3 and mTOR phosphorylation. (C) Quantification of p-mTOR/total mTOR ratio in each group. "P<0.05 vs. LV-CL. p-, phosphorylated; LV, lentivirus; CL, control.

transplantation with NSCs with STAT3 expression knocked down promotes the recovery of motor function following SCI in rats, the rats were divided into four groups. On week 8

after surgery, hindlimb weight-bearing experiments were performed in the four groups of rats. The rats in the sham group were able to use their hindlimbs to walk normally, whilst rats in the LC and SCI groups exhibited poor hindlimb strength during walking. By contrast, rats in the LS group could sometimes stand on their hind legs to support their weight and walk slowly (Fig. 3A). Results from BBB functional score curve also showed that the score in the LS group was significantly higher compared with that in the LC group from week 5 onwards after SCI (Fig. 3B). The electrophysiological restoration of SCEP responses was also subsequently explored. The SCEP waveforms in the LC and SCI group were significantly different from the SCEP waveform of the sham group, exhibiting a significantly prolonged latency and decreased amplitude. However, compared with that in the LC and SCI group, the LS group had a significantly shorter SCEP latency and significantly greater amplitude (Fig. 3C-E). Taken together, these results suggest that transplantation with STAT3-knockdown NSCs promoted functional and neurological recovery in rats following SCI.

Transplantation of STAT3-RNAi-transfected NSCs enhances tissue repair following SCI. To further clarify if the transplantation of NSCs transfected with STAT3 shRNA promotes the regeneration of damaged tissues and nerves following SCI, H\&E staining was performed to investigate the degree of tissue repair in each group. The size of the lesions was calculated 
A

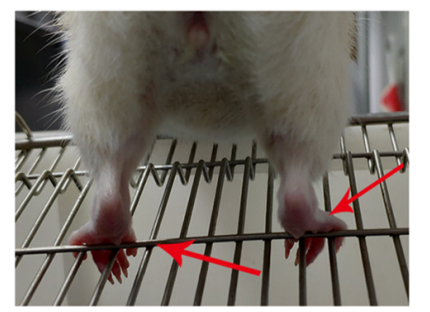

SHAM

B
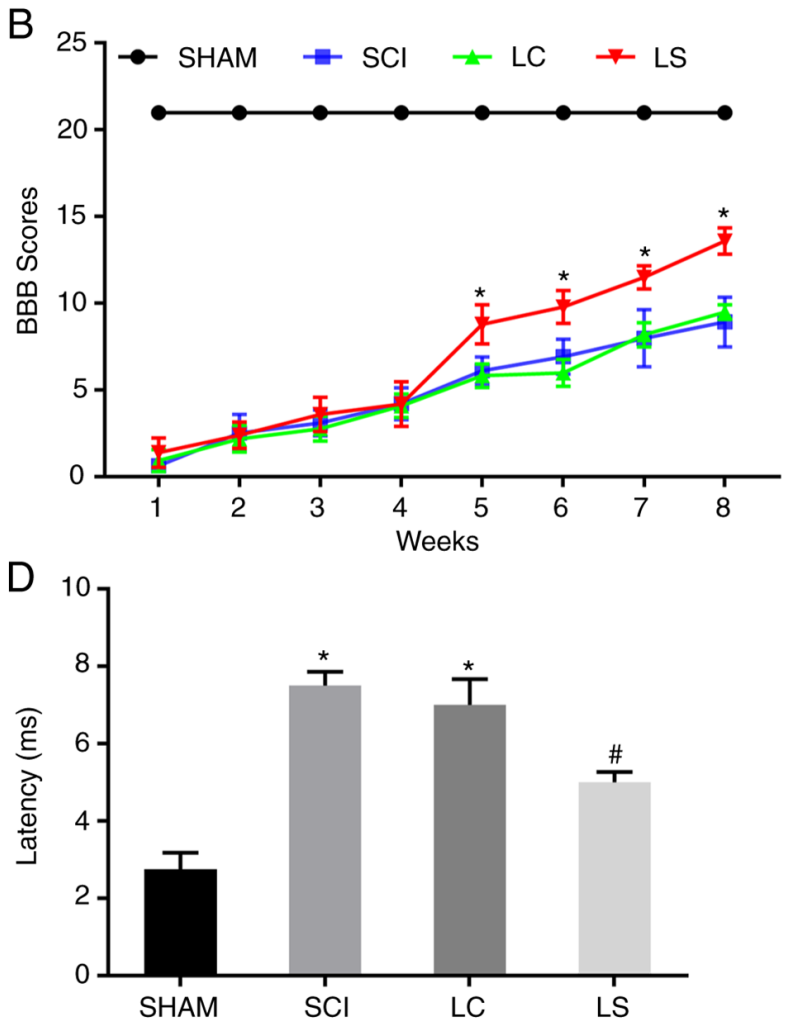

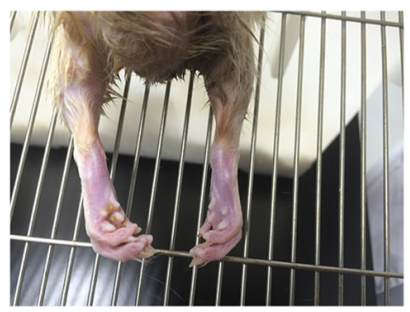

$\mathrm{SCl}$

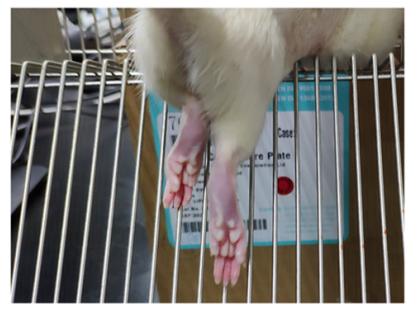

LC

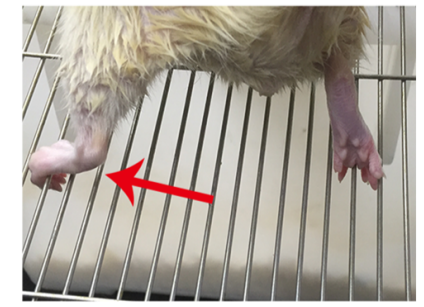

LS

C

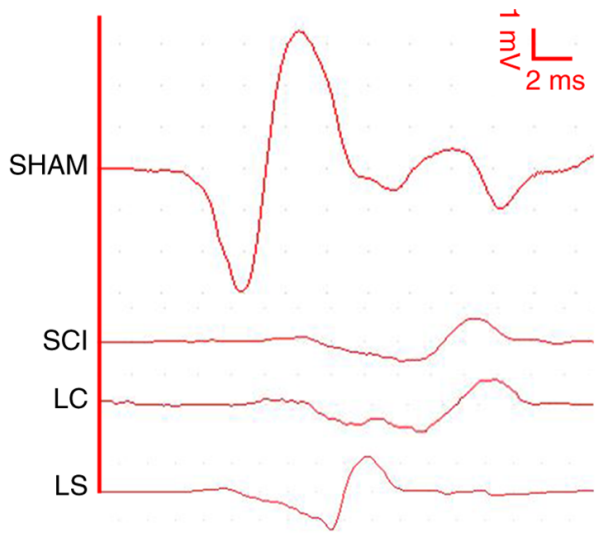

E

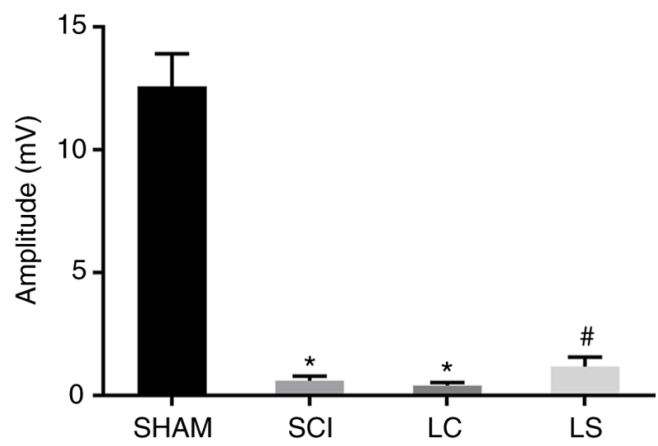

Figure 3. Transplantation of STAT3-RNAi-transfected NSCs enhances functional recovery following SCI. (A) Images showing hindlimb movements in the sham, SCI, LC and LS groups. Red arrows indicate weight-supported stepping. (B) BBB scores in the sham, SCI, LC and LS groups. (C) Electrophysiological outcomes of the SCEP recordings at 8th week after SCI. Amplitude: The magnitude of the voltage from each group of peaks to each group of baselines. Latency: The period of time from the start of the stimulation to the appearance of the peak. Quantification of the (D) latency and (E) amplitude of the SCEP. ${ }^{\text {"P }}<0.05$ vs. SHAM; " $\mathrm{P}<0.05$ vs. LC. RNAi, RNA interference; NSCs, neural stem cells; SCI, spinal cord injury; BBB, Basso, Beattie, Bresnahan; SCEP, spinal cord-evoked potential; SHAM, rats without transfection of the spinal cord; SCI, rats with complete transection of the spinal cord; LC, LV-control shRNA-transfected NSCs were injected into rats with SCI; LS, LV-STAT3 shRNA-transfected NSCs were injected into rats with SCI.

in $\mathrm{H} \& \mathrm{E}$-stained sections 8 weeks after injury. In the sagittal plane, the LS group exhibited a significantly smaller lesion area compared with that the LC group (Fig. 4A and B). To further observe the survival of neurons around the SCI area, Nissl staining was performed on the spinal cord tissue of each group. At $3 \mathrm{~mm}$ from the center of the SCI area, extensive loss of neurons was observed in the LC group. By contrast, the LS group had significantly more neurons compared with that in the LC group (Fig. 4A and C). To explore the interconnection of nerves in the injured area, a retrograde tracking experiment with FG was performed. Tissues from the LS group exhibited more neurons labeled by FG compared with those in the LC group (Fig. 4A and D). Furthermore, at the T7 spinal cord segment, FG-labeled neurons were more frequently observed in the LS group compared with those in the LC group, indicating that the LS group had more nerve connections on both ends of the lesion site (Fig. 4A and D).
Targeted inhibition of STAT3 enhances the neuronal differentiation of transplanted NSCs in spinal cord lesions. The degree of differentiation in the grafted cells was analyzed in the lesion site by immunofluorescence 8 weeks after transplantation. In the LC and SCI group, the majority of cells expressed astrocytic maker GFAP in the injured area, whilst only a small number of cells expressed neuronal markers $\beta 3$-tubulin and MAP2 (Fig. 5A and B). However, in the LS group, the percentage of $\beta 3$-tubulin positive and MAP2 positive cells was significantly higher compared with that in the LC and SCI groups, whereas the percentage of GFAP positive cells was significantly lower compared with that in the LC and SCI groups (Fig. 5A and B).

\section{Discussion}

It was shown in the present study that inhibiting STAT3 not only promoted NSC differentiation into neurons, but also 
A
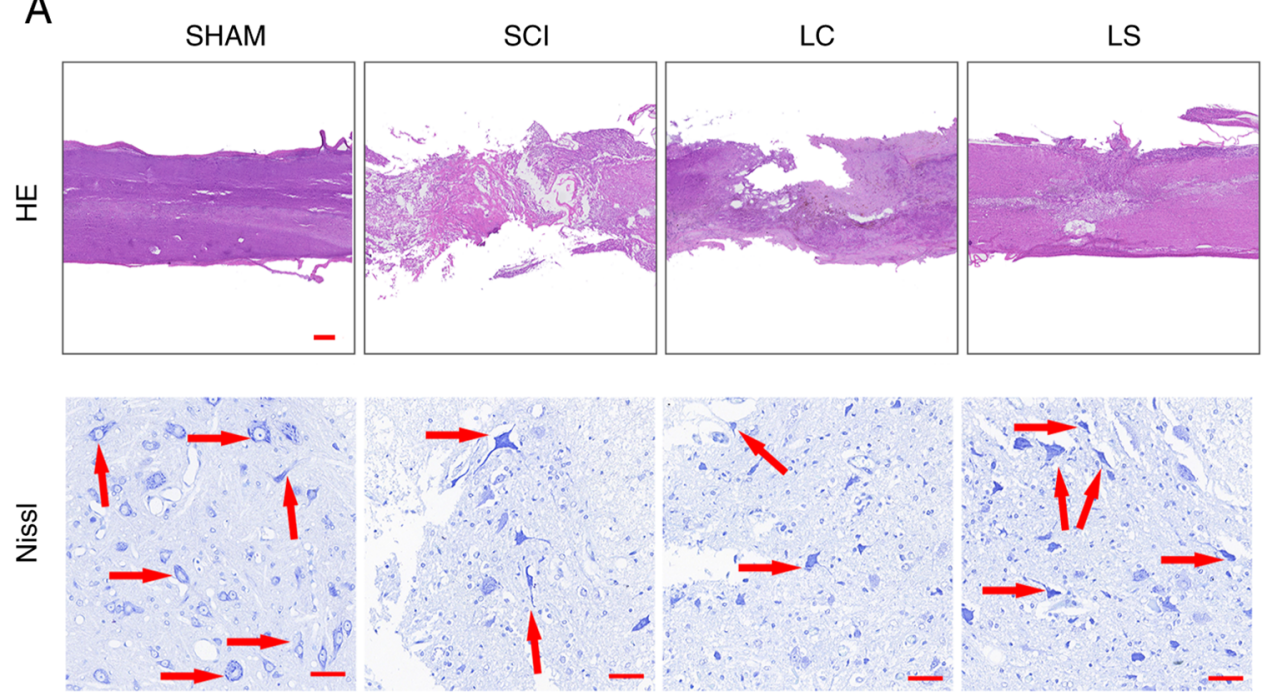

$\underset{\amalg}{\longleftarrow}$
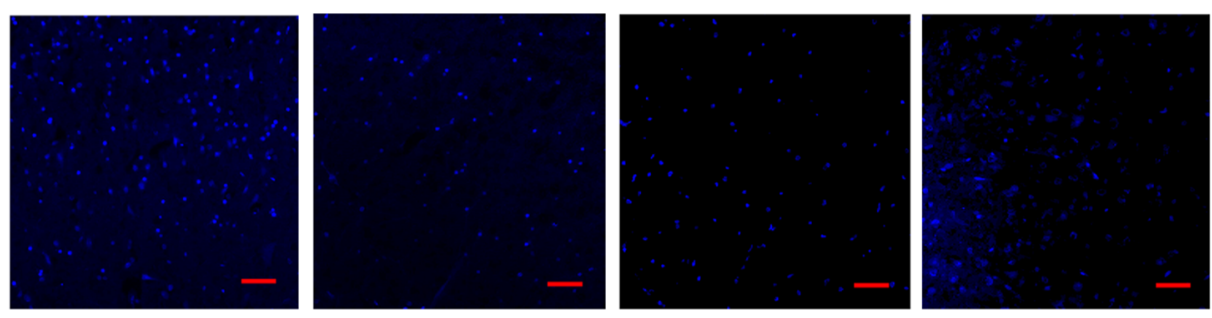

B

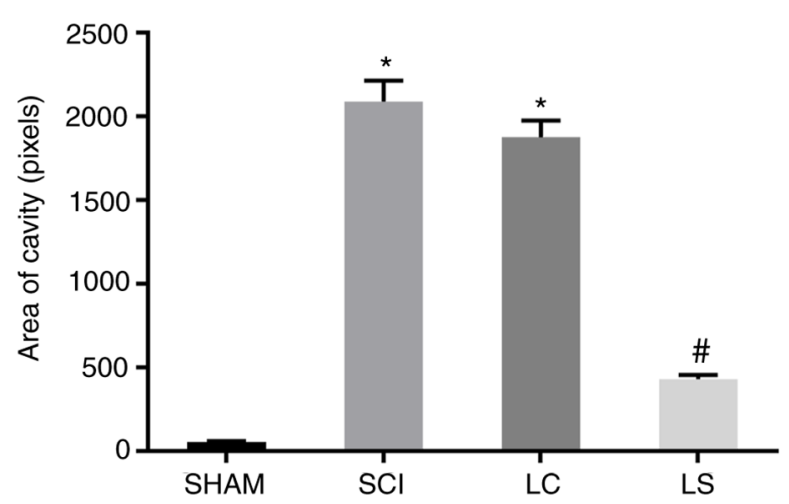

C

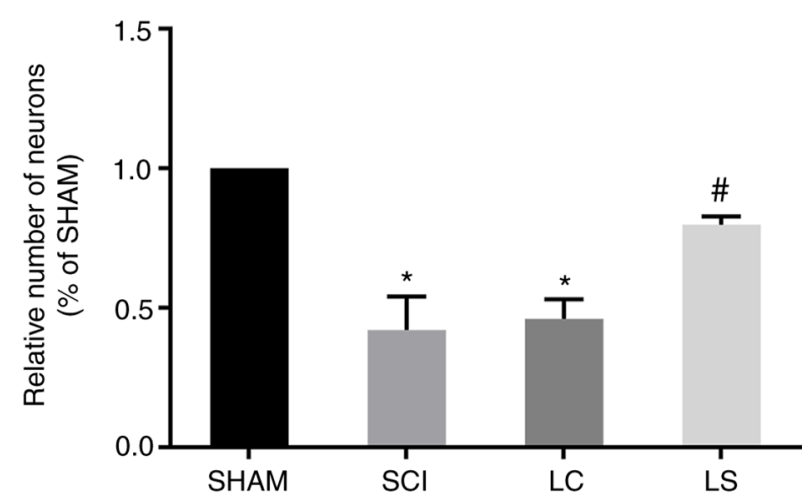

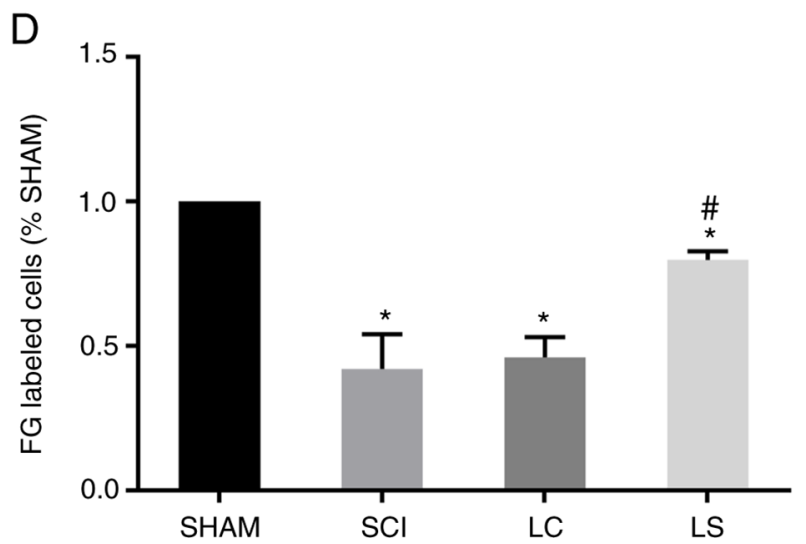

Figure 4. Transplantation of STAT3-RNAi-transfected NSCs enhance tissue repair following SCI. (A) Representative H\&E staining micrographs showing cavity formation in the sham, SCI, LC and LS groups 8 weeks after injury ( $\mathrm{n}=5)$. Images of surviving neurons $3 \mathrm{~mm}$ rostral to the injured epicenter, as shown by Nissl staining. Red arrows point to surviving neurons. FG-labeled neurons in the ventricolumna of the T7 segment spinal cord. (B) Comparison of cavity area from part (A) in each group. (C) Relative number of FG-labeled neurons in each group, which was normalized to that in the sham group. (D) Quantification analysis of the number of FG-labeled cells in each group, which was normalized to that in the sham group. "P<0.05 vs. sham, ${ }^{\text {"P }}<0.05$ vs. LC. Scale bar, $100 \mu \mathrm{m}$. RNAi, RNA interference; NSCs, neural stem cells; SCI, spinal cord injury; H\&E, hematoxylin and eosin; FG, FluoroGold; SHAM, rats without transfection of the spinal cord; SCI, rats with complete transection of the spinal cord; LC, LV-control shRNA-transfected NSCs were injected into rats with SCI; LS, LV-STAT3 shRNA-transfected NSCs were injected into rats with SCI. 


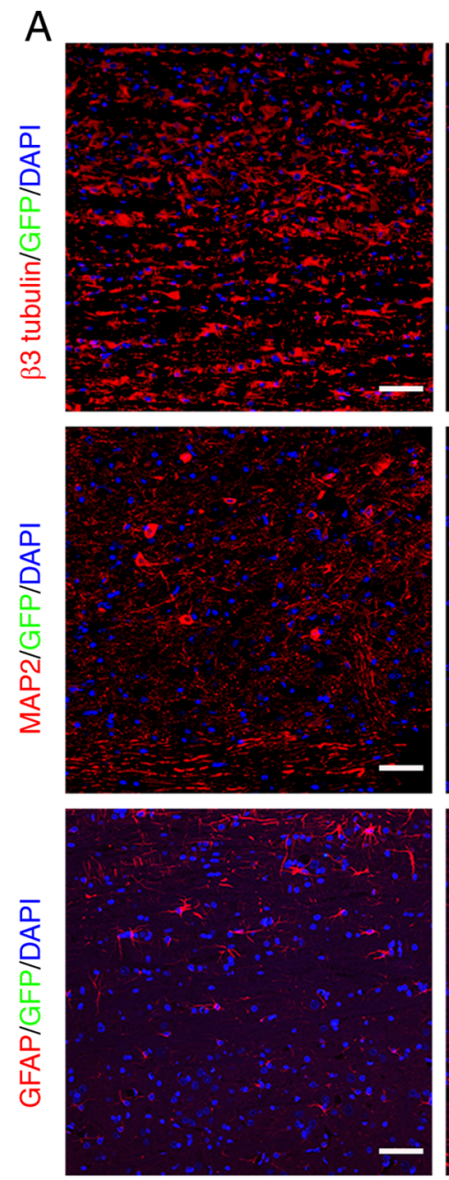

SHAM
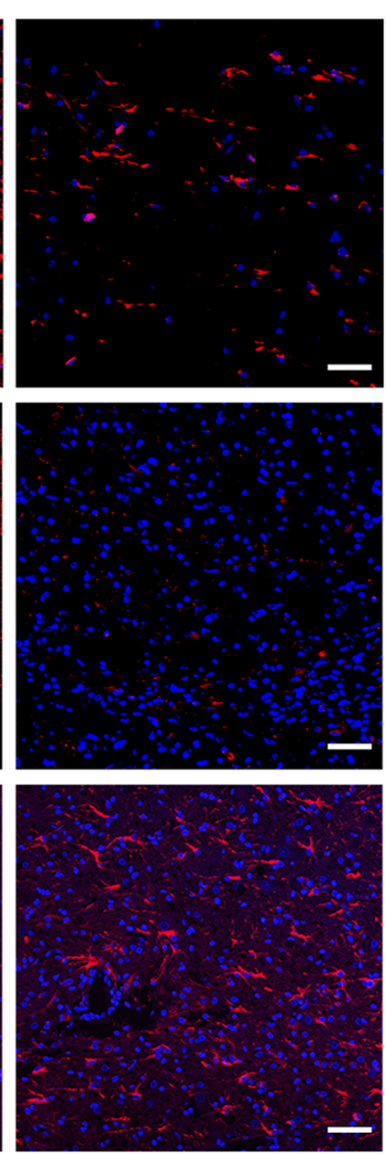

$\mathrm{SCl}$
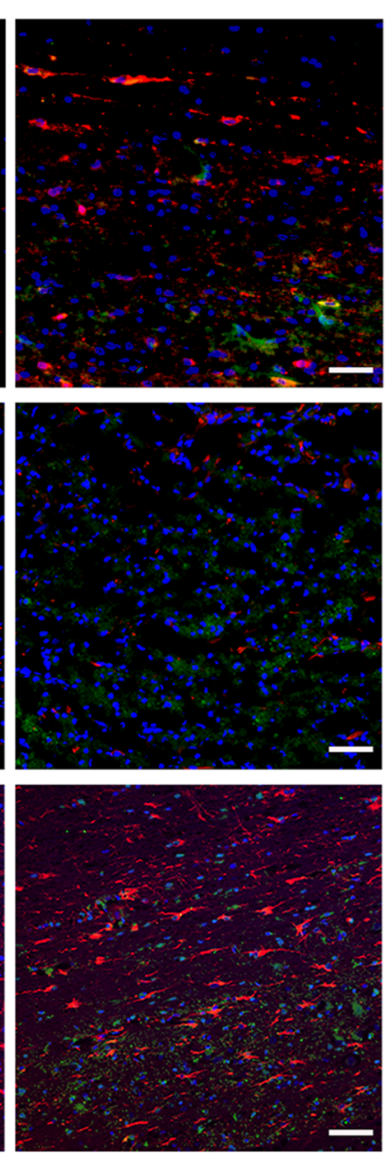

LC
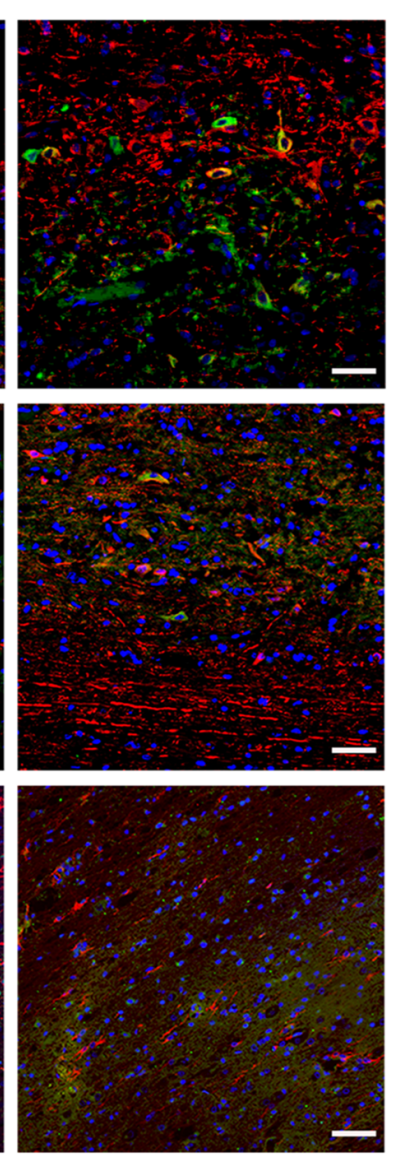

LS

B

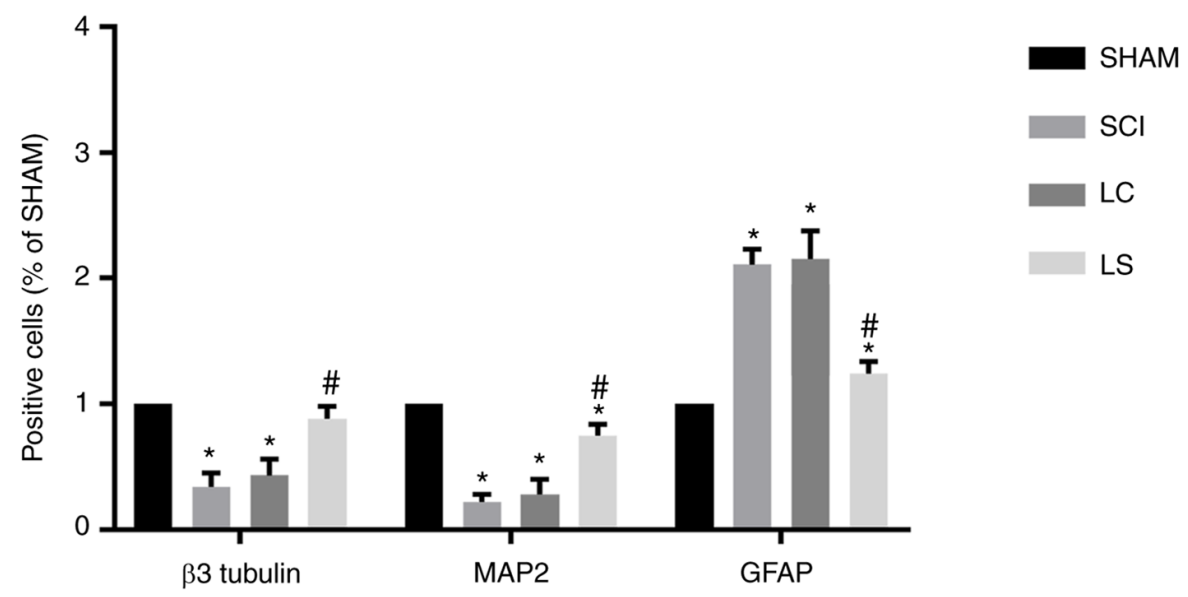

Figure 5. Targeted inhibition of STAT3 enhances neuronal differentiation of transplanted NSCs in the spinal cord lesions. (A) Immunostaining for $\beta 3$-tubulin, MAP2 and GFAP (red), GFP (green) and DAPI (blue) showing the differentiation of the transplanted NSCs in the spinal cord lesions of each group. (B) Quantification analysis of the number of $\beta 3$-tubulin-, MAP2- and GFAP-positive cells in each group, which was normalized to that in the SHAM group. ${ }^{*} \mathrm{P}<0.05$ vs. SHAM, ${ }^{\text {} P}<0.05$ vs. LC. Scale bar, $100 \mu \mathrm{m}$. NSCs, neural stem cells; MAP2, microtubule-associated protein 2; GFAP, fibrillary acidic protein; GFP, green fluorescent protein; SHAM, rats without transfection of the spinal cord; SCI, rats with complete transection of the spinal cord; LC, LV-control shRNA-transfected NSCs were injected into rats with SCI; LS, LV-STAT3 shRNA-transfected NSCs were injected into rats with SCI.

inhibited their differentiation into astrocytes, potentially through mTOR activation. In addition, it was found that the transplantation of STAT3-RNAi-transfeced NSCs into rats following SCI improved functional recovery, promoted axonal regeneration and inhibited astrocyte differentiation after SCI.
Following SCI, the limited regenerative capacity of the adult mammalian spinal cord has been attributed to the formation of cavities and glial scars that interrupt the ascending and descending pathways (26-28). NSC transplantation is now considered to be a promising method for treating SCI (29). However, compared with NSCs in the brain, most NSCs in the 
spinal cord differentiate into astrocytes, where no neurogenesis has been observed following SCI (30). STAT3 is a member of the JAK-STAT signaling family (31), which transduces signals for a number of cytokines and growth factors, including IL-6, ciliary neurotrophic factor, leukemia inhibitory factor, EGF and transforming growth factor $\alpha$, which have been implicated as triggers of reactive astrogliosis (32). STAT3 has also been found to be expressed in NSCs, such that STAT3 is activated to promote NSC proliferation but inhibit neurogenesis $(33,34)$. The binding of STAT3 to the GFAP promoter is essential for astrocyte differentiation $(35,36)$. Blocking STAT3 has been reported to favor motor neurons whilst inhibiting GFAP-positive astrocyte differentiation in NSCs in vitro (14). In the present study, STAT3 expression was inhibited in NSCs in vitro, which led to increased neuronal differentiation and reduced astrocyte differentiation at the same time. This result was consistent with previous reports $(14,33,34)$ and once again demonstrated that inhibiting the expression of STAT3 promoted the differentiation of NSCs into neurons whilst simultaneously inhibiting their differentiation into astrocytes. Of note, increased activation of mTOR was observed when the STAT3 expression was suppressed. mTOR is a serine/threonine kinase of the PI3K signaling pathway with two divergent complexes, mTOR complex 1 (mTORC1) and mTORC2 (37). mTOR serves a relevant role in the control of homeostasis of different compartments containing stem cells, including NSCs (38). A number of studies have reported that activation of the JAK/PI3K/Akt/mTOR signaling pathway promotes neuronal differentiation in NSCs (39). However, one study also showed that activating STAT3 and mTOR at the same time can promote differentiation of NSC into glial cells (40). Easley et al (41) revealed that cells with high mTORC1 activity can severely alter dendrite formation and synaptic integration. Although the downstream consequences of higher mTORC2 levels remain unclear, it is thought to be associated with cytoskeletal remodeling (37). In addition, the serine-threonine kinase FK506-binding protein/mTOR/STAT3 pathway also serves a crucial role in glial differentiation (42). Therefore, the correlation between STAT3 and the mTOR signaling pathway during NSC differentiation requires further investigation.

STAT3 is activated following SCI and plays a vital role in the differentiation and organization of astrocytes (43), which contribute to glial scar formation $(22,38,39)$. In addition, according to previous studies, the microenvironment surrounding the spinal cord lesion site following SCI result in the differentiation of exogenous NSCs into astrocytes $(28,44,45)$. This leads to the formation of glial scars in the lesion site that are widely regarded to inhibit axon regeneration and functional recovery during the chronic phase of SCI $(17,46,47)$. Dai et al (24) reported that STAT3-knockout mice exhibit less scar formation following SCI. By contrast, STAT3 inhibitors have also been shown to reverse the inhibitory effects of STAT3 on neuronal recovery (14). According to the present study, less astrocytic differentiation was observed in STAT3-RNAi-transfected NSCs, suggestive of reduced glial scar formation. In addition, compared with that in control-RNAi-transfected NSCs, STAT3-RNAi- transfected NSCs were more likely to differentiate into neurons and exhibited more dendrite outgrowth. Neurons in the SCI area of the LS group formed more connections with the upstream and downstream neurons of the SCI area, which was demonstrated by the existence of an increased number of FG-labeled neurons. Increased neuronal connectivity is considered to be one of the conditions that promote functional recovery following SCI (48). These results suggested that the STAT3-RNAi-transfected NSCs produced highly developed neurons that served a potentially useful role in neurogenesis, consistent with the results of previous studies $(14,23)$. Wu et al (49) used microRNA-15a to downregulate STAT3 in mice following SCI, following which a superior functional recovery was observed. In the present study, a more significant increase in the BBB scores of rats transplanted with STAT3-RNAi- transfected NSCs was observed, with an $\sim 50 \%$ increase in the BBB scores in that group, compared with the scores in the LC group 5 weeks following SCI. This result is superior compared with that reported by previous studies that only transplanted NSC $(45,50,51)$. Furthermore, rats transplanted with STAT3-RNAi-transfected NSCs tended to have higher SCEP amplitudes compared with rats in the LC and SCI groups, suggesting that motor and sensory axonal conduction was reinforced following transplantation with STAT3-RNAi- transfected NSCs (52). H\&E staining also confirmed that transplanting STAT3-RNAi-transfected NSCs significantly reduced the lesion volume, potentially contributing to functional recovery following SCI (53). However, although these results indicated a potentially superior recovery following the transplantation of STAT3-RNAi-transfected NSCs in SCI rats, the specific mechanism underlying these phenomena require further investigation. There are possible risks to this methodology, including the low survival rate of transplanted cells (54) and the risk of the transplanted undifferentiated stem cells developing into teratomas (55). Therefore, additional experiments are required to further confirm and clarify the safety aspects before it can be translated into other species.

In conclusion, in the present study, in vivo and in vitro evidence of the role of STAT3 as a negative regulator of neuronal differentiation in NSCs was provided. The transplantation of STAT3-inhibited NSCs appears to be a promising strategy for enhancing the benefit of NSC-mediated regenerative cell therapy for SCI, where discovery of a prominent role of STAT3 in NSC biology can enhance the understanding of NSC differentiation.

\section{Acknowledgements}

Not applicable.

\section{Funding}

The present study was supported by the Medical Scientific Research Foundation of Guangdong Province of China (grant no. A2017207), Science and Technology Promotion Program of Air Force Medical Center, PLA (grant no. 2020KTA01) and Natural Science Foundation of Guangdong Province (grant no. 2020A1515010306).

\section{Availability of data and materials}

The datasets used and/or analyzed during the current study are available from the corresponding author on reasonable request. 


\section{Authors' contributions}

LW, YW and SL designed and supervised the study. ZP analyzed the data. TL, XZ and JD conducted the study, collected the data and wrote the manuscript. SC and $\mathrm{KZ}$ collected and analyzed the data. All authors have read and approved the final manuscript. LW and TL confirm the authenticity of all the raw data.

\section{Ethics approval and consent to participate}

All experimental animal procedures were approved by the Care and Use Committee of Sun Yat-sen University (approval no. SYXX2012-0081) and performed following the Guide to the Care and Use of Experimental Animals provided by the National Research Council (1996, USA).

\section{Patient consent for publication}

Not applicable.

\section{Competing interests}

The authors declare that they have no competing interests.

\section{References}

1. Fan B, Wei Z, Yao X, Shi G, Cheng X, Zhou X, Zhou H, Ning G, Kong $X$ and Feng S: Microenvironment imbalance of spinal cord injury. Cell Transplant 27: 853-866, 2018.

2. McDonald JW and Sadowsky C: Spinal-Cord injury. Lancet 359: 417-425, 2002.

3. Dominguez E, Rivat C, Pommier B, Mauborgne A and Pohl M: JAK/STAT3 pathway is activated in spinal cord microglia after peripheral nerve injury and contributes to neuropathic pain development in rat. J Neurochem 107: 50-60, 2008.

4. Shibata N, Kakita A, Takahashi H, Ihara Y, Nobukuni K, Fujimura H, Sakoda S, Sasaki S, Iwata M, Morikawa S, et al: Activation of signal transducer and activator of transcription- 3 in the spinal cord of sporadic amyotrophic lateral sclerosis patients. Neurodegener Dis 6: 118-126, 2009.

5. LeComte MD, Shimada IS, Sherwin C and Spees JL: Notch1-STAT3-ETBR signaling axis controls reactive astrocyte proliferation after brain injury. Proc Natl Acad Sci USA 112: 8726-8731, 2015.

6. Okada S, Nakamura M, Katoh H, Miyao T, Shimazaki T, Ishii K, Yamane J, Yoshimura A, Iwamoto Y, Toyama Y and Okano H Conditional ablation of stat 3 or socs 3 discloses a dual role for reactive astrocytes after spinal cord injury. Nat Med 12: 829-834, 2006

7. Tsuda M, Kohro Y, Yano T, Tsujikawa T, Kitano J, Tozaki-Saitoh H, Koyanagi S, Ohdo S, Ji RR, Salter MW and Inoue K: JAK-STAT3 pathway regulates spinal astrocyte proliferation and neuropathic pain maintenance in rats. Brain 134: 1127-1139, 2011.

8. Park KW, Lin CY, Benveniste EN and Lee YS: Mitochondrial STAT3 is negatively regulated by SOCS3 and upregulated after spinal cord injury. Exp Neurol 284: 98-105, 2016.

9. Gu F, Hata R, Ma YJ, Tanaka J, Mitsuda N, Kumon Y, Hanakawa Y, Hashimoto K, Nakajima K and Sakanaka M: Suppression of stat 3 promotes neurogenesis in cultured neural stem cells. J Neurosci Res 81: 163-171, 2005.

10. Cao F, Hata R, Zhu P, Nakashiro Ki and Sakanaka M: Conditional deletion of stat 3 promotes neurogenesis and inhibits astrogliogenesis in neural stem cells. Biochem Biophys Res Commun 394: 843-847, 2010.

11. Gong Z, Xia K, Xu A, Yu C, Wang C, Zhu J, Huang X, Chen Q, Li F and Liang C: Stem cell transplantation: A promising therapy for spinal cord injury. Curr Stem Cell Res Ther 15: 321-331, 2019.

12. Assinck P, Duncan GJ, Hilton BJ, Plemel JR and Tetzlaff W: Cell transplantation therapy for spinal cord injury. Nat Neurosci 20: 637-647, 2017.

13. Muheremu AJ, Peng J and Ao Q: Stem cell based therapies for spinal cord injury. Tissue Cell 48: 328-333, 2016.
14. Natarajan R, Singal V, Benes R, Gao J, Chan H, Chen H, Yu Y, Zhou J and Wu P: STAT3 modulation to enhance motor neuron differentiation in human neural stem cells. PLoS One 9: e100405, 2014.

15. White CW III, Fan X, Maynard JC, Wheatley EG, Bieri G, Couthouis J, Burlingame AL and Villeda SA: Age-Related loss of neural stem cell O-GlcNAc promotes a glial fate switch through STAT3 activation. Proc Natl Acad Sci USA 117: 22214-22224, 2020.

16. Lizee G, Aerts JL, Gonzales MI, Chinnasamy N, Morgan RA and Topalian SL: Real-Time quantitative reverse transcriptase-polymerase chain reaction as a method for determining lentiviral vector titers and measuring transgene expression. Hum Gene Ther 14: 497-507, 2003

17. Chen N, Cen JS, Wang J, Qin G, Long L, Wang L, Wei F, Xiang Q, Deng DY and Wan Y: Targeted inhibition of leucine-rich repeat and immunoglobulin domain-containing protein 1 in transplanted neural stem cells promotes neuronal differentiation and functional recovery in rats subjected to spinal cord injury. Crit Care Med 44: e146-e157, 2016.

18. Livak KJ and Schmittgen TD: Analysis of relative gene expression data using real-time quantitative PCR and the 2(-Delta Delta C(T)) method. Methods 25: 402-408, 2001.

19. Bloomsmith MA, Perlman, JE Hutchinson E and Sharpless M: Behavioral management programs to promote laboratory animal welfare, In: Management of Animal Care and Use Programs in Research, Education, and Testing. 2nd edition. Weichbrod RH, Thompson GAH and Norton JN (eds). CRC Press/Taylor \& Francis, Boca Raton, FL, pp63-82, 2018.

20. Peng Z, Li X, Fu M, Zhu K, Long L, Zhao X, Chen Q, Deng DY and Wan Y: Inhibition of notch1 signaling promotes neuronal differentiation and improves functional recovery in spinal cord injury through suppressing the activation of ras homolog family member A. J Neurochem 150: 709-722, 2019.

21. Basso DM, Beattie MS and Bresnahan JC: A sensitive and reliable locomotor rating scale for open field testing in rats. J Neurotrauma 12: 1-21, 1995.

22. Zhao X, Peng Z, Long L, Chen N, Zheng H, Deng DY and Wan Y: Lentiviral vector delivery of short hairpin RNA to NgR1 promotes nerve regeneration and locomotor recovery in injured rat spinal cord. Sci Rep 8: 5447, 2018.

23. Cheng X, Yeung PK, Zhong K, Zilundu PL, Zhou Land Chung SK: Astrocytic endothelin-1 overexpression promotes neural progenitor cells proliferation and differentiation into astrocytes via the Jak2/Stat3 pathway after stroke. J Neuroinflammation 16: 227, 2019.

24. Dai J, Xu LJ, Han GD, Sun HL, Zhu GT, Jiang HT, Yu GY and Tang XM: MicroRNA-125b promotes the regeneration and repair of spinal cord injury through regulation of JAK/STAT pathway. Eur Rev Med Pharmacol Sci 22: 582-589, 2018.

25. Lee DY: Roles of mTOR signaling in brain development. Exp Neurobiol 24: 177-185, 2015.

26. Pfyffer D, Huber E, Sutter R, Curt A and Freund P: Tissue bridges predict recovery after traumatic and ischemic thoracic spinal cord injury. Neurology 93: e1550-e1560, 2019.

27. Zhang T, Liu C and Chi L: Suppression of miR-10a-5p in bone marrow mesenchymal stem cells enhances the therapeutic effect on spinal cord injury via BDNF. Neurosci 714: 134562, 2020.

28. Qian D, Li L, Rong Y, Liu W, Wang Q, Zhou Z, Gu C, Huang Y, Zhao X, Chen J, et al: Blocking notch signal pathway suppresses the activation of neurotoxic A1 astrocytes after spinal cord injury. Cell Cycle 18: 3010-3029, 2019.

29. Mothe AJ and Tator $\mathrm{CH}$ : Review of transplantation of neural stem/progenitor cells for spinal cord injury. Int J Dev Neurosci 31: 701-713, 2013.

30. Namiki J and Tator CH: Cell proliferation and nestin expression in the ependyma of the adult rat spinal cord after injury. J Neuropathol Exp Neurol 58: 489-498, 1999.

31. Decker T and Kovarik P: Transcription factor activity of STAT proteins: Structural requirements and regulation by phosphorylation and interacting proteins. Cell Mol Life Sci 55: 1535-1546, 1999.

32. Herrmann JE, Imura T, Song B, Qi J, Ao Y, Nguyen TK, Korsak RA, Takeda K, Akira S and Sofroniew MV: STAT3 is a critical regulator of astrogliosis and scar formation after spinal cord injury. J Neurosci 28: 7231-7243, 2008.

33. Ohta S, Misawa A, Fukaya R, Inoue S, Kanemura Y, Okano H, Kawakami Y and Toda M: Macrophage migration inhibitory factor (MIF) promotes cell survival and proliferation of neural stem/progenitor cells. J Cell Sci 125: 3210-3220, 2012. 
34. Kong X, Gong Z, Zhang L, Sun X, Ou Z, Xu B, Huang J, Long D, He X, Lin X, et al: JAK2/STAT3 signaling mediates IL-6-inhibited neurogenesis of neural stem cells through DNA demethylation/methylation. Brain Behav Immun 79: 159-173, 2019.

35. Cheng PY, Lin YP, Chen YL, Lee YC, Tai CC, Wang YT, Chen YJ, Kao CF and Yu J: Interplay between SIN3A and STAT3 mediates chromatin conformational changes and GFAP expression during cellular differentiation. PLoS One 6: e22018, 2011.

36. Takizawa T, Nakashima K, Namihira M, Ochiai W, Uemura A, Yanagisawa M, Fujita N, Nakao M and Taga T: DNA methylation is a critical cell-intrinsic determinant of astrocyte differentiation in the fetal brain. Dev Cell 1: 749-758, 2001.

37. LiCausi F and Hartman NW: Role of mTOR complexes in neurogenesis. Int J Mol Sci 19: 1544, 2018.

38. Russell RC, Fang C and Guan KL: An emerging role for TOR signaling in mammalian tissue and stem cell physiology. Development 138: 3343-3356, 2011.

39. Lee JE, Lim MS, Park JH, Park CH and Koh HC: S6K promotes dopaminergic neuronal differentiation through $\mathrm{PI} 3 \mathrm{~K} / \mathrm{Akt} / \mathrm{mTOR}$-dependent signaling pathways in human neural stem cells. Mol Neurobiol 53: 3771-3782, 2016.

40. Wang B, Xiao Z, Chen B, Han J, Gao Y, Zhang J, Zhao W, Wang X and Dai J: Nogo-66 promotes the differentiation of neural progenitors into astroglial lineage cells through mTOR-STAT3 pathway. PLoS One 3: e1856, 2008.

41. Easley CA IV, Ben-Yehudah A, Redinger CJ, Oliver SL, Varum ST, Eisinger VM, Carlisle DL, Donovan PJ and Schatten GP: mTOR-Mediated activation of p70 S6K induces differentiation of pluripotent human embryonic stem cells. Cell Reprogram 12: 263-273, 2010.

42. Rajan P, Panchision DM, Newell LF and McKay RD: BMPs signal alternately through a SMAD or FRAP-STAT pathway to regulate fate choice in CNS stem cells. J Cell Biol 161: 911-921, 2003.

43. Wanner IB, Anderson MA, Song B, Levine J, Fernandez A, Gray-Thompson Z, Ao Y and Sofroniew MV: Glial scar borders are formed by newly proliferated, elongated astrocytes that interact to corral inflammatory and fibrotic cells via STAT3-dependent mechanisms after spinal cord injury. J Neurosci 33: 12870-12886, 2013.

44. Kim C, Kim HJ, Lee H, Lee H, Lee SJ, Lee ST, Yang SR and Chung CK: Mesenchymal stem cell transplantation promotes functional recovery through MMP2/STAT3 related astrogliosis after spinal cord injury. Int J Stem Cells 12: 331-339, 2019.

45. Hackett AR, Lee DH, Dawood A, Rodriguez M, Funk L, Tsoulfas P and Lee JK: STAT3 and SOCS3 regulate NG2 cell proliferation and differentiation after contusive spinal cord injury. Neurobiol Dis 89: 10-22, 2016.

46. Hosseini SM, Sani M, Haider KH, Dorvash M, Ziaee SM, Karimi A and Namavar MR: Concomitant use of mesenchymal stem cells and neural stem cells for treatment of spinal cord injury: A combo cell therapy approach. Neurosci Lett 668: 138-146, 2018.
47. Lopez-Serrano C, Torres-Espín A, Hernández J, Alvarez-Palomo AB, Requena J, Gasull X, Edel MJ and Navarro X: Effects of the post-spinal cord injury microenvironment on the differentiation capacity of human neural stem cells derived from induced pluripotent stem cells. Cell Transplant 25: 1833-1852, 2016.

48. Squair JW, West CR, Popok D, Assinck P, Liu J, Tetzlaff W and Krassioukov AV: High thoracic contusion model for the investigation of cardiovascular function after spinal cord injury. J Neurotrauma 34: 671-684, 2017.

49. Wu WD, Wang LH, Wei NX, Kong DH, Shao G, Zhang SR and Du YS: MicroRNA-15a inhibits inflammatory response and apoptosis after spinal cord injury via targeting STAT3. Eur Rev Med Pharmacol Sci 23: 9189-9198, 2019.

50. van Gorp S, Leerink M, Kakinohana O, Platoshyn O, Santucci C, Galik J, Joosten EA, Hruska-Plochan M, Goldberg D, Marsala S, et al: Amelioration of motor/sensory dysfunction and spasticity in a rat model of acute lumbar spinal cord injury by human neural stem cell transplantation. Stem Cell Res Ther 4: 57, 2013.

51. Zhao XM, He XY, Liu J, Xu Y, Xu FF, Tan YX, Zhang ZB and Wang TH: Neural stem cell transplantation improves locomotor function in spinal cord transection rats associated with nerve regeneration and IGF-1 R expression. Cell Transplant 28: 1197-1211, 2019.

52. Winkler T, Sharma HS, Gordh T, Badgaiyan RD, Stålberg E and Westman J: Topical application of dynorphin A (1-17) antiserum attenuates trauma induced alterations in spinal cord evoked potentials, microvascular permeability disturbances, edema formation and cell injury: An experimental study in the rat using electrophysiological and morphological approaches. Amino Acids 23: 273-281, 2002.

53. Ramadan WS, Abdel-Hamid GA, Al-Karim S and Abbas AT: Histological, immunohistochemical and ultrastructural study of secondary compressed spinal cord injury in a rat model. Folia Histochem Cytobiol 55: 11-20, 2017.

54. Pereira IM, Marote A, Salgado AJ and Silva NA: Filling the gap: Neural stem cells as a promising therapy for spinal cord injury. Pharmaceuticals (Basel) 29: 65, 2019.

55. Trounson A and McDonald C: Stem cell therapies in clinical trials: Progress and challenges. Cell Stem Cell 17: 11-22, 2015.

This work is licensed under a Creative Commons Attribution-NonCommercial-NoDerivatives 4.0 International (CC BY-NC-ND 4.0) License. 